\title{
Foreword
}

\section{The National Cancer Institute Early Detection Research Network: The Associate Membership Program}

\author{
Padma Maruvada and Sudhir Srivastava* \\ Cancer Biomarkers Research Group, National Cancer Institute, Bethesda, MD 20892, USA
}

Tremendous progress has been made in the war against cancer on many fronts, including biology, diagnosis, treatment and monitoring of cancer in the recent years. Despite this progress cancer remains a second leading cause of death. There is growing enthusiasm for early detection of cancers. Technological inventions allow us to identify molecular events that will effectively detect cancer earlier in biological specimens. This approach enables us to develop molecular biomarkers for detecting cancer early. It is believed that such a molecular marker approach may not only enable the detection of the disease earlier, but may also improve earlier therapeutic interventions and better survival and quality of life.

Biomarkers are poised to play a critical role in meeting the expectations of cancer patients by providing clinical tests that can detect these biomarkers in biological specimens that can be obtained by non-invasive means. For molecular detection, it is necessary to understand the biological nature of carcinogenic transformation and genetic and molecular changes leading to preneoplastic lesions. Unfortunately not many biomarkers are available today that can be successfully applied into clinical settings. For the successful trans-

*Corresponding author: Sudhir Srivastava, Ph.D., MPH, Chief, Cancer Biomarkers Research Group, National Institutes of Health, Executive Plaza North, Room 3142, 6130 Executive Boulevard; Rockville, MD 20852-7346, USA. Tel.: +1 301496 3983; Fax: +1 301402 8990; E-mail: srivasts@mail.nih.gov. lation of the biological innovations, it is necessary for them to be systematically evaluated before they can be applied in the clinic.

Successful development of biomarkers depends not only on the approaches to understanding the biology of carcinogenesis, but also on the application of stateof-the science technological approaches for detecting changes that occur at cellular, genetic, molecular and protein levels. With the completion of sequencing of the human genome, various molecular approaches, including genomic, proteomic, epigenomic methods have accelerated in the discovery and development of molecular markers for the detection of cancer. Scientists in the Early Detection Research Network (EDRN) are committed to translating cancer biomarkers research into clinical applications and for creating the needed infrastructure for validating biomarkers. Initiated in 2000, the Associate Membership Program has complemented the resources and expertise of EDRN and brought a new focus to emerging research areas that were not adequately covered by the Network. The recently held workshop of the associate members program enabled the EDRN investigators with needed collaborative support, and also served as a complementary forum for exchanging research ideas with other EDRN investigators.

The Cancer Biomarkers Research Group, Division of Cancer Prevention, National Cancer Institute, sponsored a one-day workshop of the EDRN Associate Members on June 13th, 2004 in Bethesda, MD. Asso- 
ciate members addressed various aspects of preclinical cancer detection and presented their research supported through the Associate Membership Program. Another major goal of this workshop was to improve interactions among current EDRN associate members and to foster cooperation and interaction with the Network. A few selected articles submitted by associate members are published in this special issue of
Cancer Biomarkers, Section A of Disease Markers. These articles highlight the accomplishments of and types of research pursued through the Associate Member Program.

The Editor-in-Chief would like to express his sincere appreciation of Dr. Jacob Kagan and Mr. Donald Johnsey for soliciting, reviewing and editing the abstracts. 